attacks is the most important feature of the therapy. The relationship of upper respiratory tract infection to the catarrhal state must be emphasised, but this does not constitute the whole picture which is that of a generalised respiratory catarrh.

\section{Summary}

I. The pathogenesis and clinical features of the catarrhal child have been fully discussed.

2. The bronchoscopic findings in these children have been described.

3. The rôle of upper respiratory tract infection in the catarrhal child has been stressed.

4. A new therapeutic programme for the catarrhal child has been suggested.
5. The results of this new therapy, with reports on six illustrative cases, have been given.

\section{REFERENCES}

I. DELLER, F. C. (1946), Post-grad. med. J., 22, 43.

2. MONCRIEFF, A. (I934), "Diseases of Children," Garrod, Batten, Thursfield and Paterson, London, p. 4I3.

3. GRIFFITHS, IVOR' (1937), Lancet, ii, 723.

The great encouragement and help both academic $\frac{\bar{\sigma}}{\overline{0}}$ and practical which I have received from Mr. J. D. $\frac{\pi}{\widetilde{T}}$ McLaggan, F.R.C.S., and Mr. Ivor Griffiths, $\stackrel{\mathbb{Q}}{ }$ M.S., F.R.C.S., must not be overlooked, for without them much of the understanding of the catarrhal $\vec{\circ}$ child which I believe I have now, would have been:lost to me. Much has been learned also from my $\vec{\omega}$ anaesthetic colleagues, especially Dr. G. Hochs-s child and Dr. Woodfield Davies who have so oftenoo borne with me very patiently.

\title{
RADIOTHERAPY AND CANCER
}

\author{
By D. W. Smithers, M.D., D.M.R.
}

(Director of the Radiotherapy Department of the Royal Cancer Hospital (Free) and the Royal Free Hospital; $\overline{3}$ Radiotherapist Brompton Hospital for Diseases of the Chest, and the West End Hospital for Nervous Diseases. $)^{2}$

\section{Introduction}

Fifty years ago in November, I895, Wilhelm Conrad Röntgen discovered X-rays; within one year Henri Becquerel had discovered radioactivity, and within three years Marie and Pierre Curie had isolated radium. Röntgen, Becquerel, and the Curies opened up new fields for research and their discoveries have had the most profound influence on the progress of science. One direct outcome of their work was the introduction to medicine of a valuable new method of treatment, but there is probably no type of treatment that has such a wide application and has been used for so long which is so little understood or its value so little appreciated by the general body of medical men.

Radiotherapy is the use of certain types of ionising radiations in the attack upon disease. Its most important, though by no means only, use in medicine is in the cure and the relief of suffering due to the scourge of cancer. A scourge because, although it is a curable disease in its early stages, it is responsible for the death of more adults than any other condition, except heart disease, and because it is second to none in its capacity to produce fear and suffering.

\section{(I) The Physical Basis of Radiotherapy}

Radiation is a process of transferring energy from one place to another; radiotherapy applies this energy to living tissues which are abnormal either in structure or in function. In canogre treatment it is employed to destroy malignati cells in such a way as to give the natural defenteso of the body the maximum aid possible in dealing with the disease and in repairing the damage done

The types of radiation in general use at the present time are X-rays, produced at voltages ranging from about 50,000 to several hundred thousand volts, and the gamma rays and beta rays 3 from radium. Beta rays are particles (electrons) and $\mathrm{X}$ and gamma rays are waves. These waves. however, behave very like particles in that theire energy is concentrated in units (photons), the energy in each unit depending on the wavelength? of the radiation-the shorter the wavelength the greater the energy of the photon.

To understand the application of these various types of radiation in medicine it is necessary to study the underlying physical principles involved These radiations affect matter, both organic andE. inorganic, in fundamentally the same way. Thest produce changes in the atoms of the substance they irradiate, either by knocking electrons right out of the atoms (ionisation) or, by displacin electrons within the atoms (excitation). In either case the properties of the affected atom are change and this change may initiate a whole series o. chemical reactions. With inorganic materials the results of the irradiation may be slight, such as change of colour of the material, but with biologica? materials the changes produced may be considero 
able and the death of living cells or even of whole organisms is a frequent sequel to irradiation.

The spatial distribution of the energy absorbed varies with the type of radiation, and the method of application employed and these differences lead to considerable variations in biological effect. Thus the various radiations each have their own field of usefulness according to the condition to be treated. For example, the beta rays from radium produce their effect in the surface layers of the material irradiated but do not penetrate very far. They are therefore used for the treatment of very superficial conditions. On the other hand, X-rays produced at high. voltages have considerable penetration through the human body and are used for treating more deeply-seated lesions.

The progress in nuclear physics being made at the present time is placing new instruments in the hands of the radiotherapist. Much work remains to be done before the significance and value of these new discoveries in the treatment of cancer are fully appreciated. Neutrons, artificial radioactive substances, extremely high-voltage X-rays, and penetrating beta rays are becoming available, however, and some at least will offer new opportunities to the radiotherapist and hope for further improvement in the results obtained by radiotherapy.

\section{Atomic Structure}

An atom consists of a nucleus around which electrons circulate. The nucleus contains nearly all the weight of the atom and is positively charged. The electrons which weigh approximately $I / I, 850$ th of the weight of the lightest nucleus are negatively charged. The number of electrons circulating around the nucleus is equal to the number of positive charges on the nucleus so that they neutralise the charge on the atom.

The simplest element is hydrogen which has a nucleus composed of one positively charged particle, a proton, and one electron circulating round it (Fig. I). The next element in the periodic table (elements in order of increasing atomic weights) is helium with two electrons, a nuclear charge of two but with a weight four times that of the hydrogen atom. The helium nucleus is composed of two protons and two other particles, known as neutrons, of approximately the same weight as protons but carrying no electric charge (Fig. 2). The atoms when arranged in the periodic table and numbered have nuclei on which the charge increases by one unit as we pass from one atom to the next. The atomic number indicates the size of the nuclear charge (and therefore the number of electrons in the outer atom). Lithium with atomic number 3 has three electrons and a nucleus composed of 3 protons and 4 neutrons (as its atomic weight is

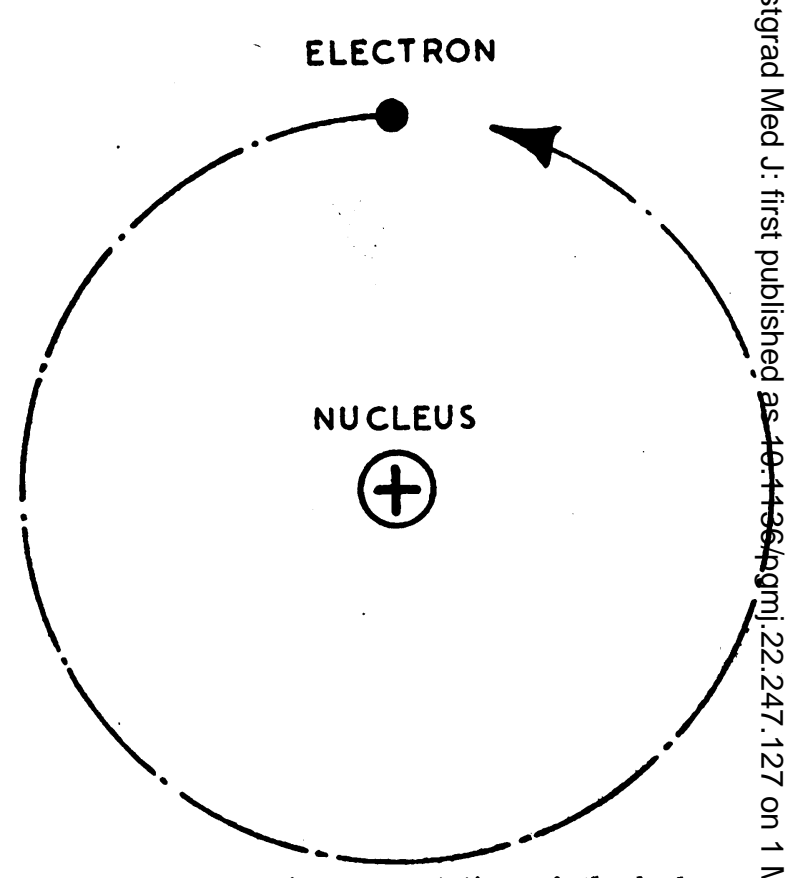

FIG. 1.-Diagrammatic representation of the hydrogen $\frac{3}{0}$ atom $\left({ }_{\mathrm{r}} \mathrm{H}^{\mathrm{x}}\right)$. The diameter of the nucleus is approximately $\frac{1}{10,000}$ of the diameter of the atom. The $\overrightarrow{0}$ mass of the electron is approximately $\frac{1}{1,850}$ of the f mass of the nucleus.

seven times that of the hydrogen atom). The number of neutrons is equal to the weight of the atom less the atomic number. All atoms of the same substance do not have the same weight, $\stackrel{\circ}{\circ}$ however, and a hydrogen atom may have a mass $\overrightarrow{\vec{P}}$ of two when its nucleus is made up not only of 3 one proton but with one neutron as well. Such atoms with different weights but the same atomic number are known as isotopes, the heavy isotopes of hydrogen (deuterium) combined with oxygen 3 . forms "heavy water." Lithium has a light isotope of mass six with three protons (atomic number 3 ) and three neutrons in the nucleus (Fig. 2).

The most important characteristic of an element is its atomic number. Elements are given chemicalo symbols and isotopes are shown by writing the figures representing the mass above and after the? symbol; the atomic number is often written before and under the symbol. The two isotopes of ${ }^{\text {II }}$ lithium would thus be written ${ }_{3} \mathrm{Li}^{6}$ and ${ }_{3} \mathrm{Li}^{7}, \widetilde{O}$ hydrogen ${ }_{1} \mathrm{H}^{1}$ and heavy hydrogen ${ }_{1} \mathrm{H}^{2}$. Atomic nuclei are not altered in chemical reactions whicho are the result of a re-arrangement of their outer electrons in such a way as to link elements together. Isotopes are difficult to separate because their $\stackrel{\infty}{?}$ chemical and physical properties are primarily dependent on their nuclear charge and although they differ in weight their nuclear charges are the same. 

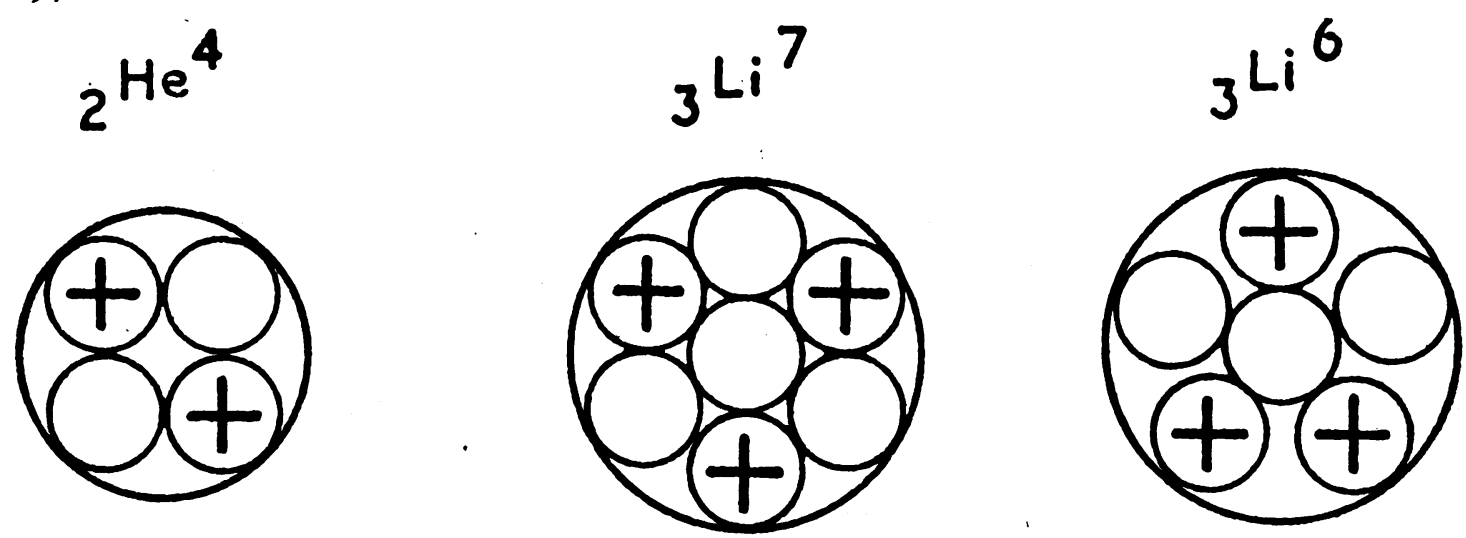

\section{O PARTICLE HELIUM NUCLEUS}

\section{ISOTOPES LITHIUM NUCLEI}

FIG. 2-Diagrammatic representation of Helium and Lithium nuclei.

The atom is a minute entity held together by a vast amount of energy which renders it highly resistant to attack. The electrons circulating round the nucleus in the atom travel in certain defined orbits (the method of wave mechanics expresses this more accurately in terms of a system of waves surrounding a centre of force). Some electrons travel in orbits close to the nucleus, others further away and others in the outer part of the atom. The heavier atoms have a large number of groups of electrons travelling in $\dot{a}$ number of different orbits (Fig. 3). Electrons in the atom can only travel in these orbits; they may jump from one orbit to another or be knocked out of the atom altogether, but they cannot take up some intermediate position inside the atom. An electron from outside may strike an electron in the atom handing over to it sufficient energy to move it from one orbit to another. Such an atom has gained energy and been altered to an unstable state. It will tend to revert to its normal state and in doing so will give off the energy gained in the form of radiation. If the energy change is large the radiation will be of short wavelength (with large quanta or units of energy-photons). Each atom has its own set of energies for its various orbits and when excited gives out radiations of specified wavelengths (characteristic radiation). A larger amount of energy is required to displace electrons from orbits close to the nucleus than from orbits in the outer atom.

\section{The Production of $X$-Rays}

An X-ray tube consists of an evacuated glass vessel containing a heated filament (the cathode) which is the source of electrons, and a metal target (the anode) which is the source of X-rays (Fig. 4). A high electrical potential is put across the tube so that the electrons from the cathode are speeded across the gap to strike the target. As these particles strike the target they are suddenly arrested and their energy of motion is transformed into electromagnetic energy (waves). The targe thus becomes a source of $\mathrm{X}$-rays, mostly due te. the alteration in motion of the electric charge when the electrons collide with the target, but also partly due to characteristic radiation resulting from the displacement of electrons from their $\mathbb{D}$ orbits in the atoms of which the target is composed.

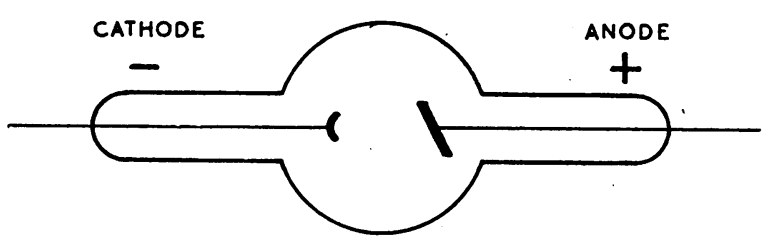

FIG. 4.-Diagrammatic representation of an X-ray tube. The cathode filament is heated by a small electric current and a high electric potential is put across the 음 tube to speed the electrons across the gap to strike the target. The sudden arrest of the electrons (cathode rays) when they strike the target results in the emission of X-rays.

Spontaneous Radioactivity

N

Some of the elements of high atomic number with the most complicated nuclear structures (e.g.0 uranium, thorium, radium) undergo spontaneous disintegration giving off radiation in the process. $\frac{\sim}{\mathbb{D}}$ These rays consist of either alpha particles (mass $\stackrel{?}{+}$ four, positive charge two-the nuclei of helium 0 atoms-Fig. 2), beta particles (negative charge ${ }^{-}$ one-electrons) or gamma rays (short wavelength $\stackrel{\odot}{\mathbb{Q}}$ 
rays) which are an accompaniment of the emission of alpha or beta particles. The gamma rays are more penetrating than the alpha and beta rays, and as they are not charged particles they are not deflected by electric or magnetic fields. When a radioactive element gives off an alpha particle it changes to a different element with an atomic number lower by two and a mass lower by four; the emission of a beta particle increases the atomic number by one but leaves the mass of the nucleus unaltered. Some radioactive elements disintegrate rapidly, others very slowly, the rate cannot be altered and is expressed as the time it would take for half the atoms initially present to disintegrate. This time (the half-life or half-value period) varies from a fraction of a second to millions of years (it is 3.82 days for radon and I,650 years for radium).

\section{Artificial Radioactivity}

Until recently the only radioactive materials known were those found naturally and their separation and purification was a very laborious and costly procedure. With new techniques and instruments almost all the common elements can now be produced in a radioactive form.

The mass of a nucleus is slightly less than the total mass of the protons and neutrons of which it is composed. It is now recognised that energy and mass are equivalent and that this slight difference in mass is due to the energy required to split up the nucleus. Radioactive substances disintegrating spontaneously give off particles from their nuclei and this emission of particles is accompanied by the release of the binding energy in the form of short wavelength radiation (gamma rays). It is possible to bombard atoms with particles so that some of them will enter the nucleus giving it increased energy and making it unstable. These nuclei will tend to revert to their normal state giving off the energy gained. This process is known as artificial radioactivity as it is induced in atoms that do not normally undergo spon? taneous disintegration. Neutrons are commonly. used for the induction of artificial radioactivity because they have no charge, do not interact with the electric field and can therefore penetrate more easily to the nucleus where charge is concentrated (Fig. 5).

\section{Particulate Radiations}

The particulate radiations obtained from radio $\overrightarrow{0}$ active substances (alpha and beta rays) have little $\overrightarrow{-}$ penetration, being stopped by thin layers of material ${ }_{\infty}^{\omega}$ Alpha rays are absorbed in a few centimetres of air or in a thin sheet of paper, and are therefores. of little use in treatment. Beta rays from radium are sometimes used for the treatment of very super ficial lesions for they are approximately IOOA times more penetrating than the alpha raysemitted, but are still absorbed by quite thin layers of material such as $5.0 \mathrm{~mm}$. of aluminium. Special apparatus is therefore necessary for the production of high-speed particles if direct use is to be made of particulate radiations for the treatment of any but very superficial tumours.

High speed neutrons can now be obtained dirêt in a form suitable for use in radiotherapy. Thet instrument which has made possible the productiono of neutrons in sufficient quantities for use in therapys is the Cyclotron (Fig. 6), an apparatus designed by Lawrence, for research work in atomic physics. It consists essentially of two hollow semi-circularo electrodes, known as Dees on account of their shape, $\overrightarrow{\overrightarrow{0}}$ which are placed between the poles of a magnet. 3 A heated wire provides a source of electrons. $\mathrm{A}^{3}$ minute quantity of heavy hydrogen gas is introduced which, when bombarded by these electrons

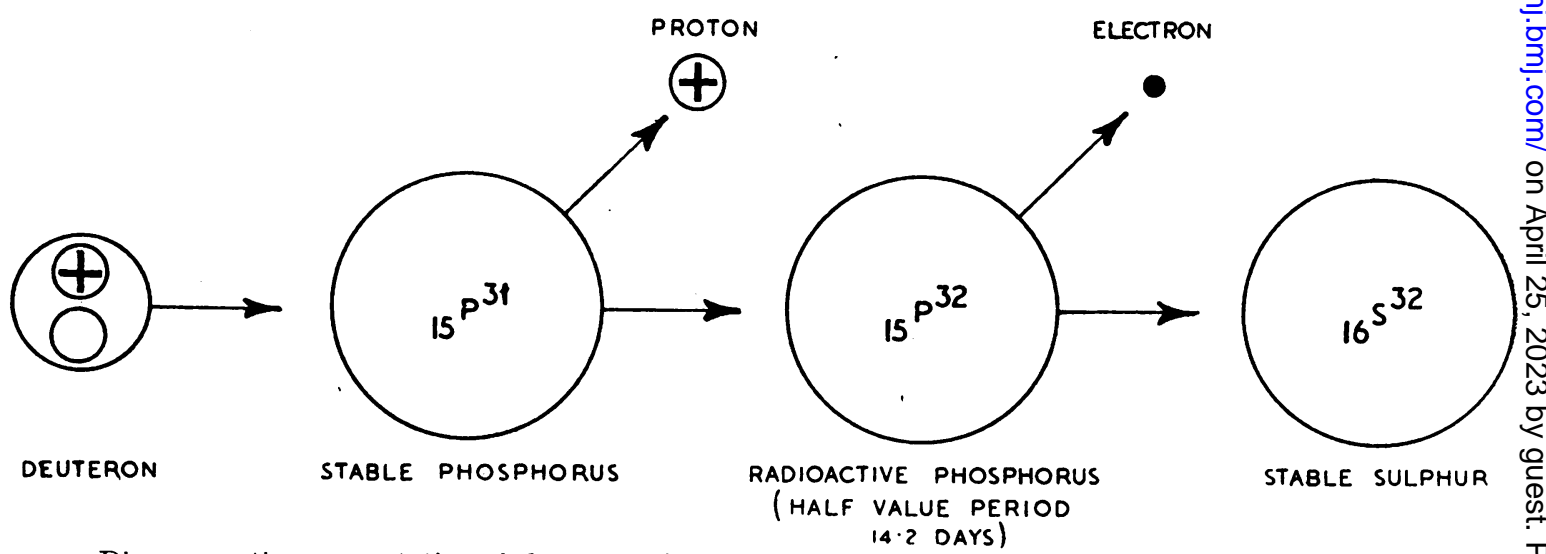

FIG. 5.-Diagrammatic representation of the production of artificially radioactive phosphorus by the bombardment of stable phosphorus with deuterons. The neutron in the deuteron penetrates the phosphorus nucleus which; then $\frac{\vec{\Phi}}{\mathrm{D}}$ becomes excited and gives off an electron so changing to stable sulphur. 


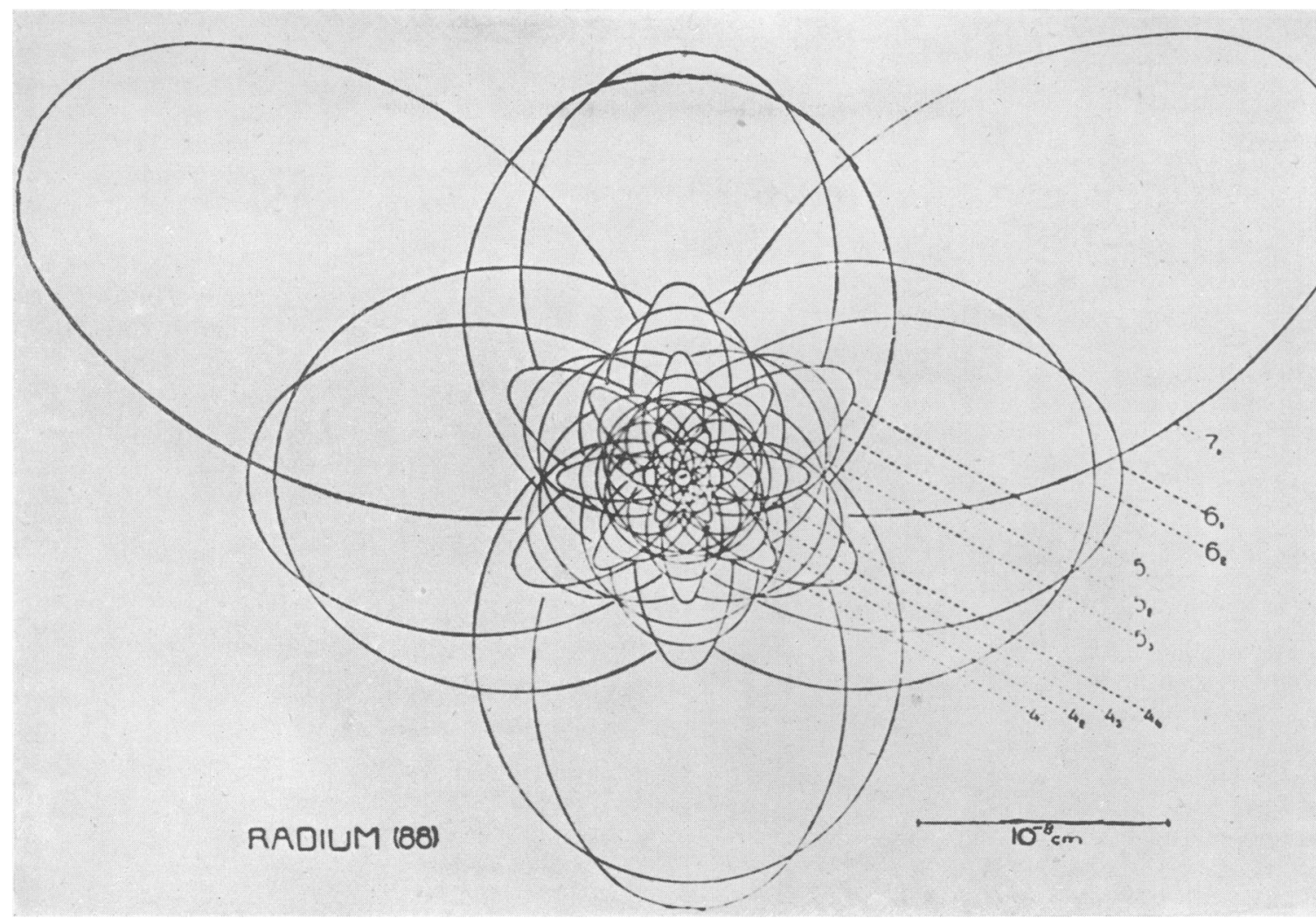

Fig. 3.-Hypothetical structure of the radium atom as suggested by Niels Bohr prior to the development of wave-mechanics.

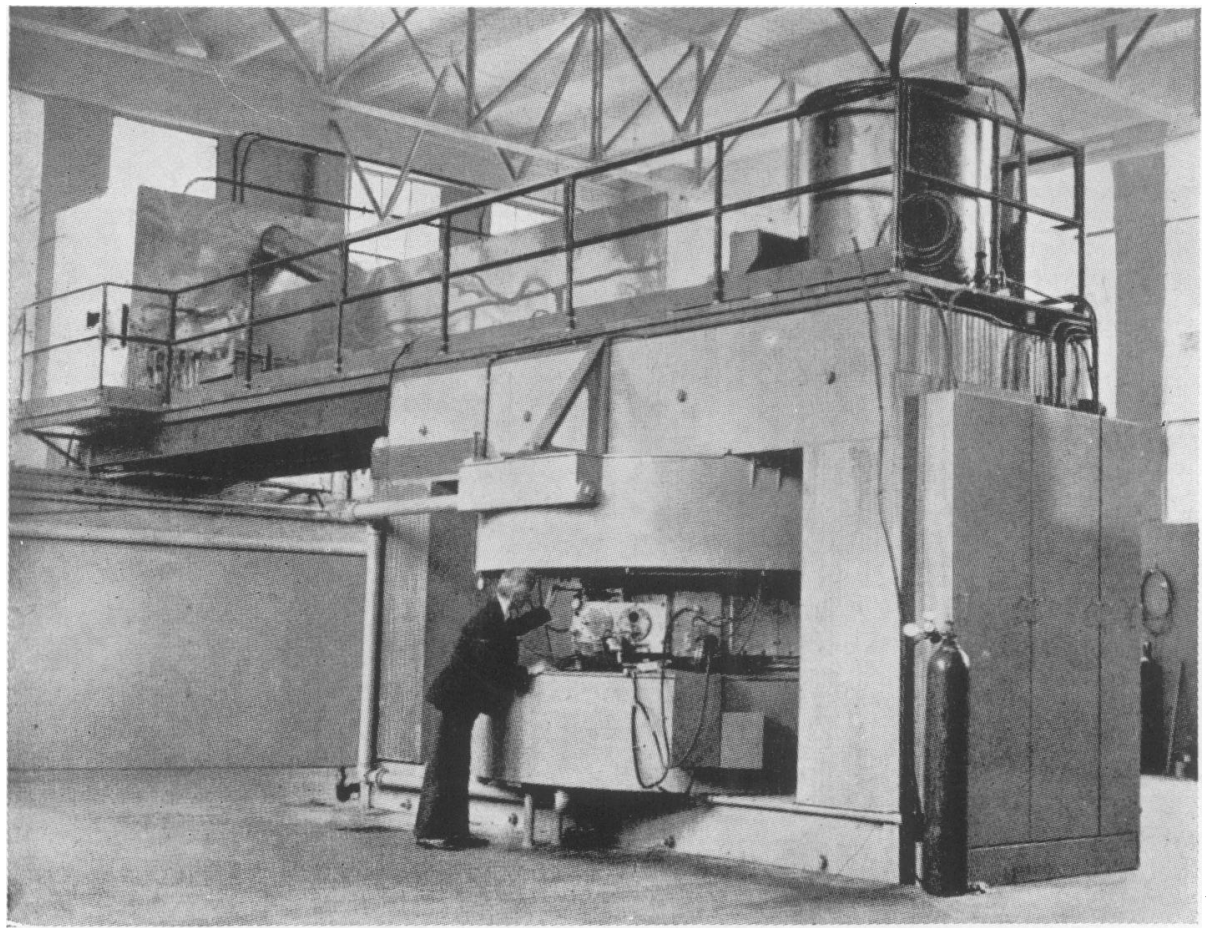




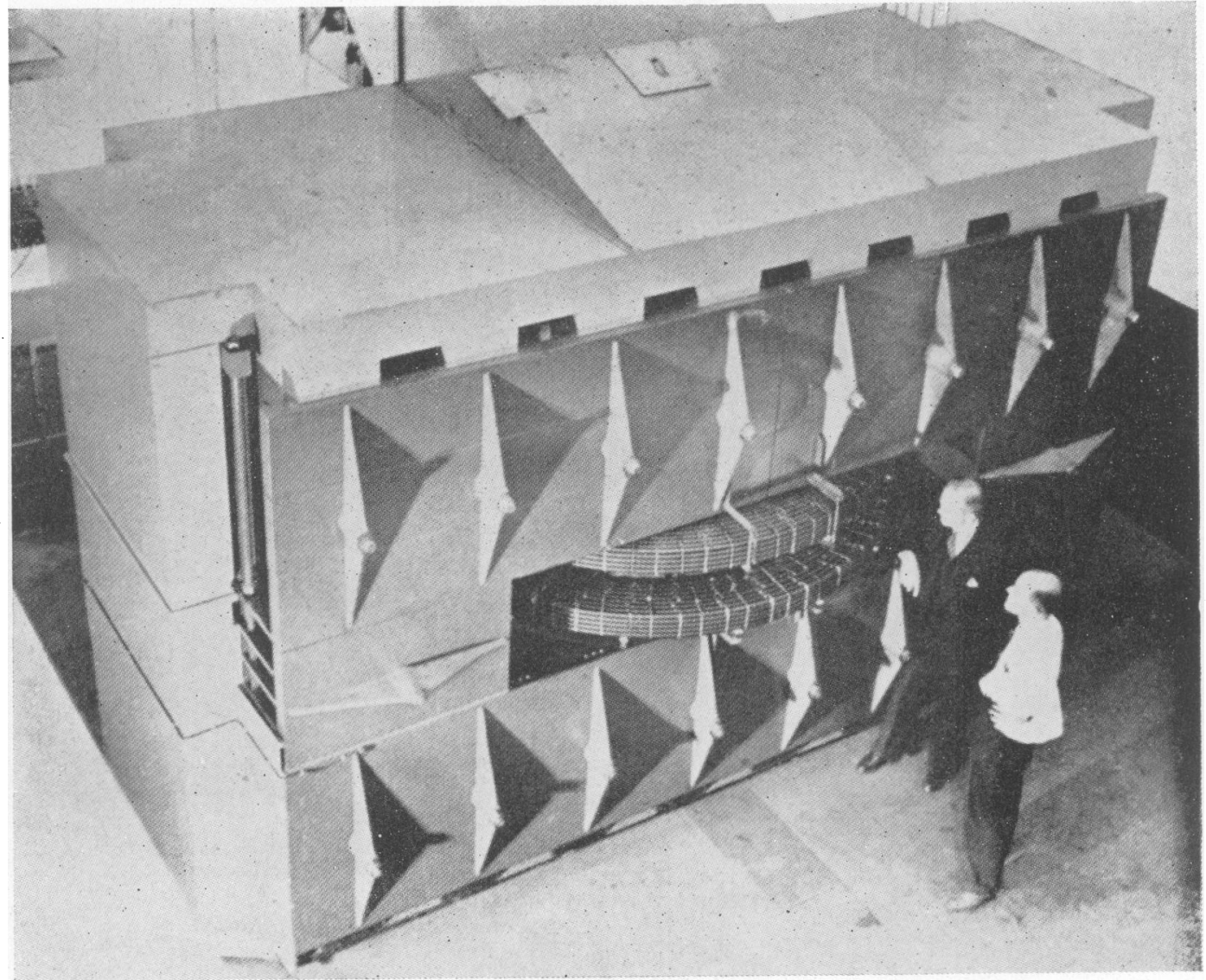

FIG. 8.-Diagram showing the degree of radiosensitivity of chromosomes during the developmental cycle in pollen grain (PG) cells. The most sensitive period is between $30-20 \mathrm{hrs}$. before metaphase and coincides with the time of chromosome reduplication.

(By courtesy of Dr. P. C. Koller.) 

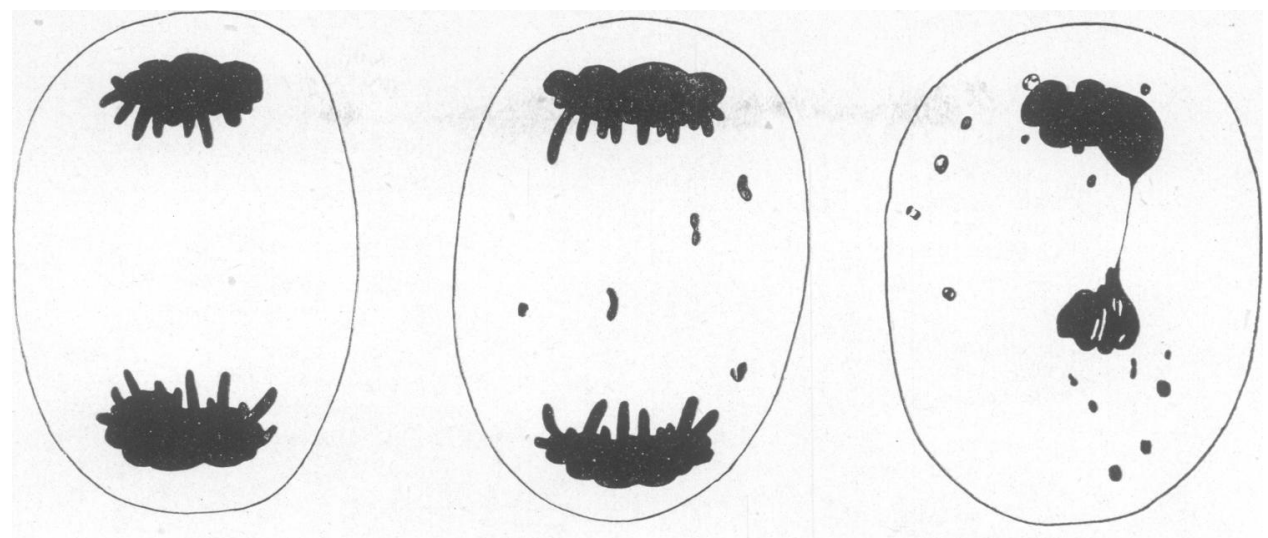

A $\quad$ B

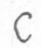

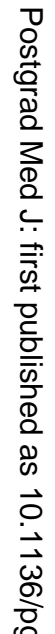

FIG. 9.-Cell division in tumour cells (A) before and (B and C) after irradiation. The radiation induced fragments 3 are scattered and lost in the cytoplasm leading to the degeneration of the two daughter cells.

(By courtesy of Dr. P. C. Koller.)

Fig. 11.-Five-gramme teleradium unit with pneumatic transference of radium to lead-lined safe.

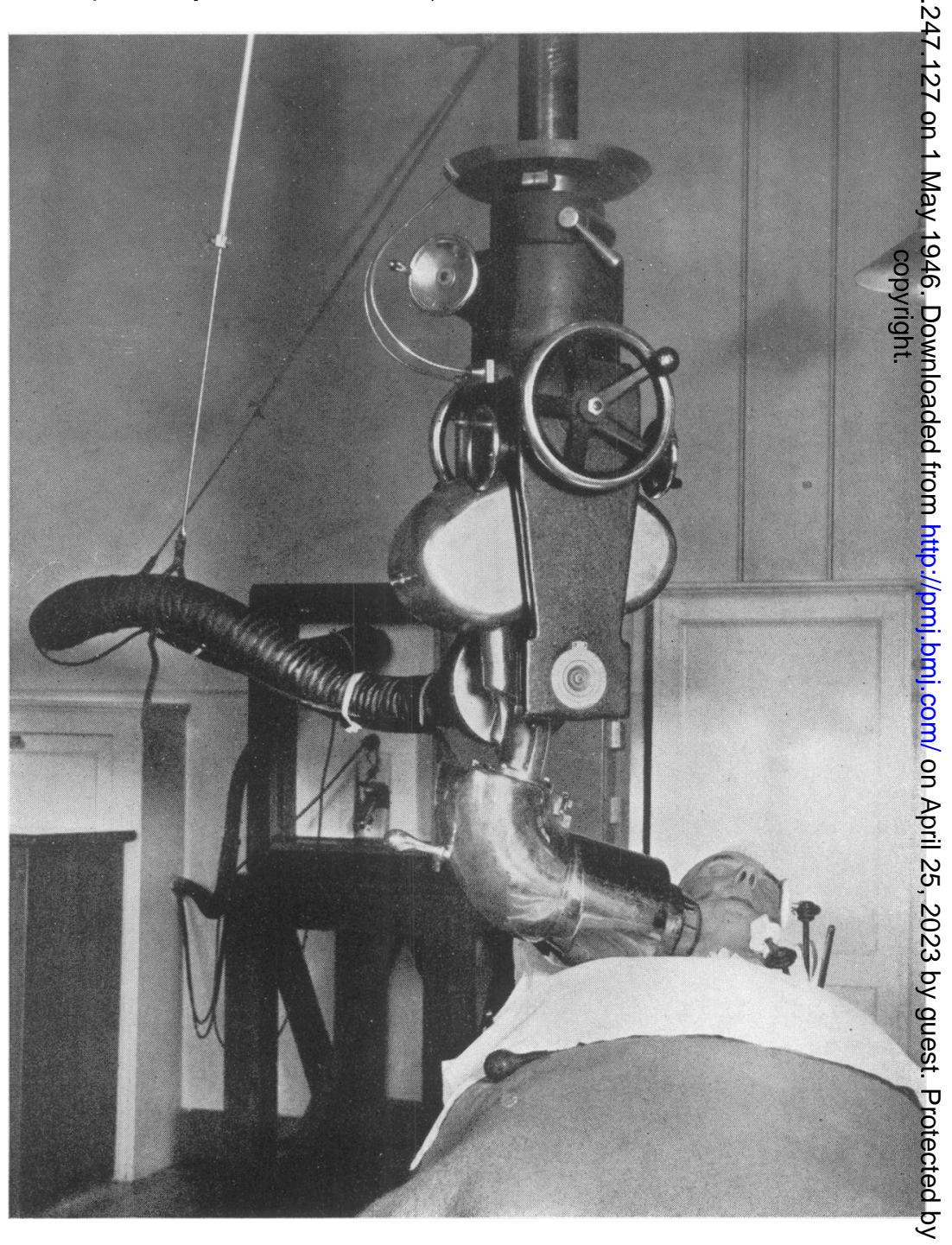




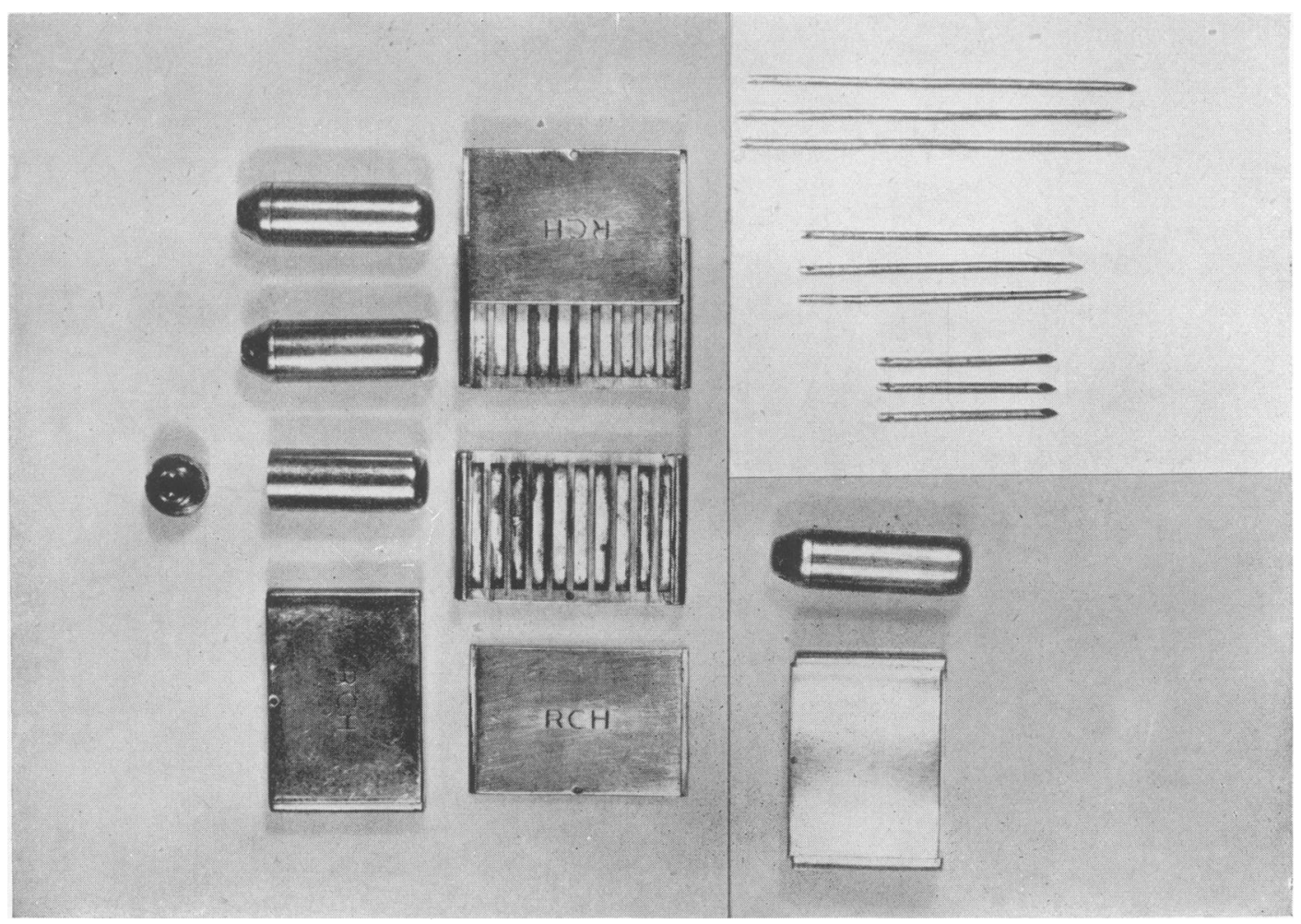

Frg. 10.-A selection of radium needles, tubes, and boxes.

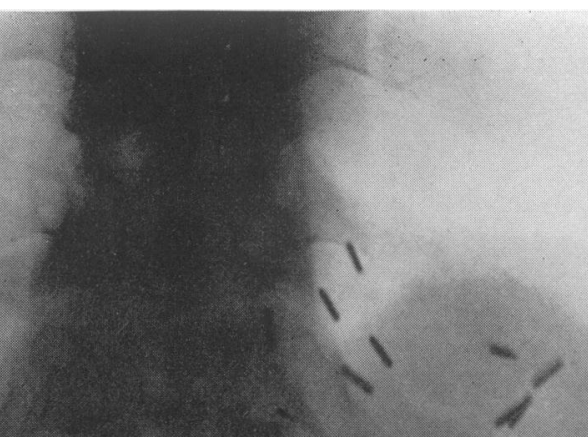




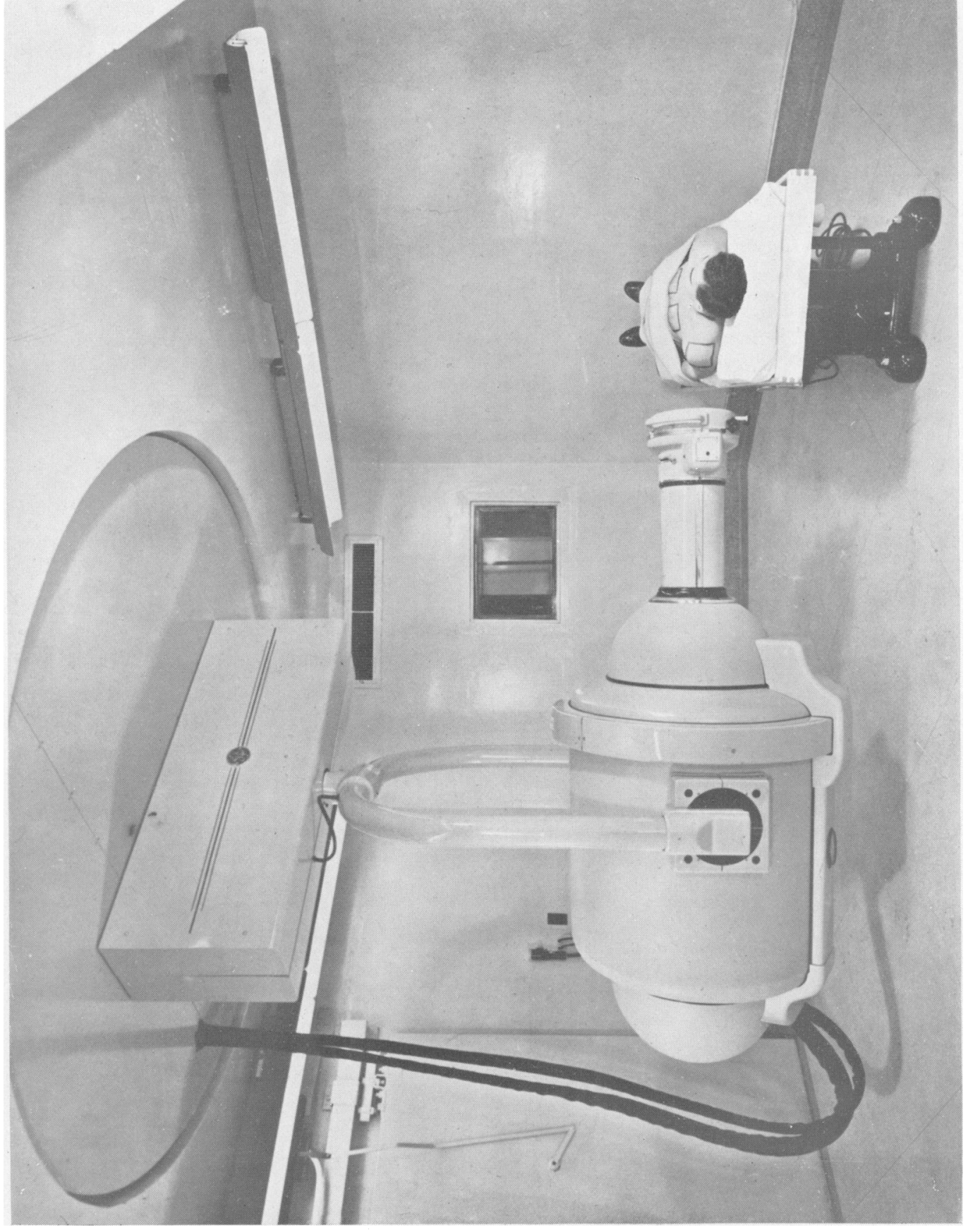


D. W. SMITHERS, M.D., D.M.R.

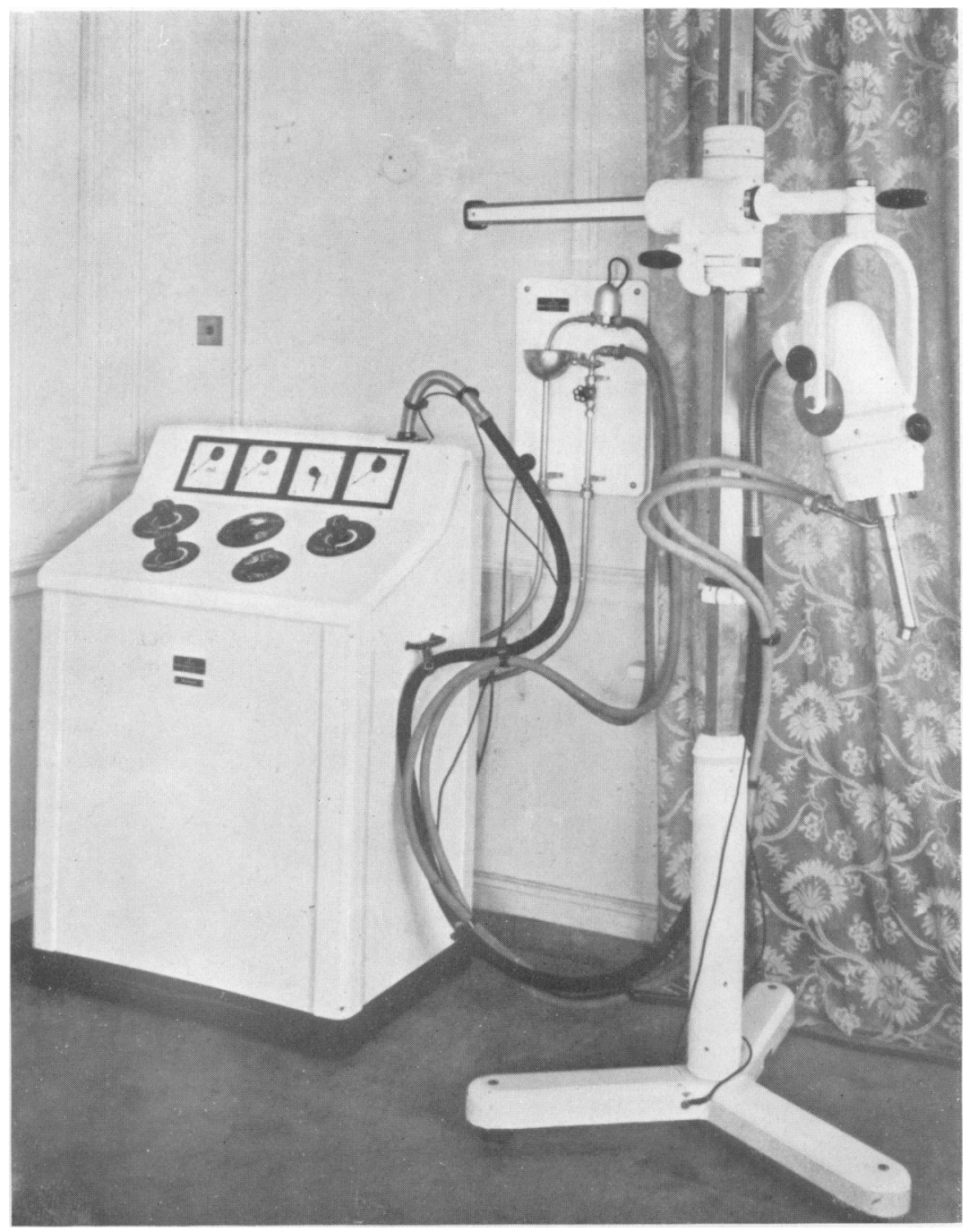

FIG. 14.-Siemens "contact" X-ray therapy plant. 
loses some of the single electrons from the outer part of the atoms leaving the heavy hydrogen nuclei (deuterons) behind. Particles in a strong magnetic field travel in circular orbits so that these deuterons circulate between the poles of the magnet inside the hollow electrodes. The deuterons are repelled by the positive Dee and attracted to the negative Dee so that they are accelerated as they pass on their circular course from one Dee to the other. An oscillating voltage is applied to the Dees so that each time particles pass from one electrode to another they are given a further acceleration. As the particles gain speed they spiral outwards taking a longer course but taking the same time to complete each circuit. The oscillating voltage is kept at a constant frequency and a stream of particles in process of being accelerated spiral from the centre outwards getting faster as they go. The stream of high speed deuterons is finally allowed to strike a beryllium target from which neutrons are then emitted. These neutrons may either be used directly for treatment or for bombardment of other substances for the purpose of inducing artificial radioactivity.

The other new instrument which will probably become of great importance in therapy is the Betatron (Fig. 7) designed by Kerst. It is rather similar to a cyclotron though the method of acceleration of the particles is different. Particles in a cyclotron can only be accelerated to a speed much less than that of light so that only heavy particles can be given great energy. The betatron can accelerate light particles (electrons) till they acquire very great energies. The electrons circulate in a doughnut-shaped vacuum tube placed between the poles of a magnet and are accelerated by magnetic induction; the electromotive force is continually applied directly to the electron stream by a time-varying magnetic field. As the electrons travel faster the magnetic field is increased so that the electrons do not spiral outwards until they have reached their maximum speed, having travelled as much as 200 miles, and special orbit expanding coils are energised. These coils supply a heavy reverse current which rapidly reduces the magnetic field and allows the electrons to spiral outwards to strike a target and produce X-rays. By this means-X-rays have been produced at the equivalent of 50 million volts and a Ioo-millionvolt Betatron has now been constructed. The beam of electrons may one day be used directly in treatment, but it is not yet certain whether this would be useful even if it were practicable.

\section{The Absorption of Energy in Tissues}

When radiation is applied to a patient part of the energy in the beam is absorbed. It is only that fraction of the energy which is absorbed that is biologically active (Grotthus' law). The energy io the particles, or in the photons of the $\mathrm{X}$ or gamma rays, is handed over to electrons in the tissues by collision. In these collisions a photon may hand over all its energy to a tissue electron removing. it from one of the inner orbits of an atom and sending it out with considerable velocity (photo $\bar{z}$ electron) the atom then reverting to its normas state emitting further radiation (characteristiw. radiation) in the process. A photon may onlop hand on part of its energy to an electron (recoip electron) and proceed on its way with changed direction, less energy and so a longer wavelengthis (modified scatter), or it may merely be deflected by an electron without loss of energy (unmodified scatter). The tissues are themselves, therefore, के source of X-rays (characteristic and scattered which in turn hand on energy to further electrons until the energy absorbed is dissipated in intense electronic activity. Electrons are made to traven in the tissues with varying speeds for varying distances according to their energy (up to a few millimetres at most under existing therapeutio conditions, but possibly up to Io centimetres or more with the Betatron) and are slowed down b collision with other electrons which they remove from molecules in their paths. A molecule that looses an electron becomes positively charges (positive ion) and an electron torn from one masecule attaches itself to another which then becoreses negatively charged (negative ion) the process be known as ionisation. It is the passage of a electron through some sensitive area or the fore mation of an ion pair at a sensitive point in the tissues, which forms the basis of the biologicat action produced by radiation.

\section{(2) The Biological Basis of Radiotherap}

Irradiation of living tissues causes damage The damage done depends on the amount and distribution of the energy absorbed, the rate of absorption during exposure, the intervals between successive exposures, and the total period over which the whole treatment is spread (the times dose relationship); the sensitivity of the differents types of cell, and the stage of mitotic activit and duration of mitotic cycle in individual cells (the inherent cell sensitivity); and the state of the surrounding tissues (sensitivity due to environment and possibilities of repair).

Radiotherapy for cancer is the application of radiation to the patient so that the energy absorbed is concentrated in the tumour, and is delivered if such a way as to do an adequate amount of damage to the tumour with the least possible damage to the normal tissues. For success this requires high degree of precision in planning and adminis 
tering the treatment both with regard to the amount and distribution of the energy absorbed and to the relationship of dose with time.

The literature on the biological actions of radiation is so immense that it has become very difficult for the research workers in this field, let alone the radiotherapists, to summarise and absorb it. The radiotherapist who wishes to apply the knowledge gained to the practical task of treating patients has the problem further complicated for him. He is not so directly concerned with the response of isolated biological material, which is the basis of most of this research work, as he is with the effects on certain tissues, forming part of a whole organism, which cannot be irradiated in isolation. In cancer, where the main tissue to be irradiated is composed of cells differing widely in development and activity and often abnormal both in structure and behaviour, the practical application of experimental findings is especially difficult. What follows is therefore an incomplete summary of the position for no authoritative account either of the relative importance of the various time-dose relationships or of the sequence of biological effects that result from irradiation applied to tumours in patients is yet possible.

\section{Structural Changes in the Cell Nucleus Resulting from Irradiation}

Much of the confusion that exists, regarding the response of living tissues to irradiation is due to the fact that many of the effects observed and measured are the results of a series of unknown reactions set in motion by the radiation. Even the mechanism by which individual cells are affected by radiation is not yet understood completely. It was shown over 30 years ago that the nucleus was the part of the cell most sensitive to radiation. There are probably many effects of radiation on the cell that have yet to be discovered, however, and it may be that important changes are produced primarily in the cytoplasm. Living tissues of any type can be killed by massive doses of radiation no doubt due to non-specific chemical changes resulting from the large amount of energy absorbed by the component cells, but lethal changes can also be produced by very small doses particularly on cells that are just about to divide. The resting nucleus has a homogeneous appearance which gives way to characteristic and complicated structure as mitotic activity commences. As Crowther wrote "the correlation between the appearance of structure and abnormal sensitivity seems too close to be fortuitous."

We have seen that the action of radiation on tissues is discontinuous; the energy absorbed is concentrated in a comparatively small number of atoms. The "target theory" of the biological action of radiation assumes that action takes $\stackrel{0}{\circ}$ place either when an electron passes through a ̊ sensitive spot or when an ion pair is formed at 3 such a spot. In I928 Muller showed that X-rays $\stackrel{\varnothing}{\circ}$ produced gene mutations, an action on minute $C$. structures situated on the chromosomes. This is $\overrightarrow{\vec{F}}$ an irreversible change dependent only on the dose given and quite independent of any time relationship. Stern in I929 produced evidence that $\frac{\sigma}{\bar{N}}$. radiation could break chromosomes. Chromosome $\frac{\vec{\nabla}}{\overrightarrow{2}}$ breakage is one of the initial, fundamental, direct $\stackrel{\varnothing}{~}$ effects of radiation on cells which can be observed $๗$ and analysed. It is a discontinuous effect $\vec{o}$ occurring at various points in the nucleus which $\overrightarrow{-}$ we have seen becomes especially sensitive to this $\vec{\omega}$ type of damage as its characteristic structure $\frac{P}{\partial}$ develops. The chief method of measuring the direct effect of radiation on cells is by assessing the frequency with which these breaks are induced $\stackrel{N}{N}$ in chromosomes. Chromosomes are most liable i to radiation induced breakage at the end of the resting stage when they divide longitudinally into $\vec{N}$ two chromatids (Fig. 8). The breaks produced may effect one or both of these sister chromatids. An induced break may rejoin in the original position, may reunite in a different way with a sister or non-sister chromatid or may remain open as a single break. Breaks that are not reconstitute in the normal way will result in abnormalities 通? the daughter cells as the damaged chromosomes. cannot migrate normally along the spindle and either chromosome fragments will be left behind or chromosome bridges will be formed (Fig. 9). In both cases the resulting cells will be deficient in chromosome material and will die.

\section{Chemical Changes Following Structural Alteration.}

Chromosome structure and cell activity are beginning to be described in relation to the chemical processes taking place in cells. In I942 Caspersson and Santesson by microscopical studies, microchemical analysis and ultra violet spectro- 3 . scopy of cells showed that intensive hyperfunction 8 of heterochromatin (a specific portion of the chromosomes concerned with protein synthesis, $ᄋ$ the basis of cell-growth) is characteristic of cancer cells. They showed that abnormal chromosome behaviour and increased division rate may be $\underset{N}{N}$ explained in terms of disturbances in this system or which controls protein synthesis. Nucleic acids 0 are the agents of this synthesis in the cell and $\underset{\mathcal{W}}{\mathrm{N}}$ Mitchell has shown that significant quantitative o changes are produced in nucleic acid metabolism by therapeutic doses of radiation. Koller, by cytological analysis of tumour cells, showed that $\stackrel{\oplus}{+}$ all their chromosome abnormalities and the in- 0 creased rate of division itself might be explained o in terms of a quantitative change in the nucleic 
acid synthesis which is assumed to be due to alteration in the heterochromatic region of the chromosomes. Radiation thus produces fundamental changes in the structure of the sensitive nuclei of cells as a result of which the chemical cell processes, particularly those concerned with growth, are altered.

\section{Effects of Variation in Dose-Time Relationship on Cell Reaction}

With irreversible actions such as the production. of gene mutations the change is independent of time and depends only on the amount of energy absorbed. With chromosome abnormalities, however, the time relationship of dosage is important. The number of cells showing chromosome abnormalities after irradiation at low dosage-rates is less than at high dosage-rates (Koller). Cell recovery (reunion of the induced chromosome breaks in the same position) is favoured by delay in producing a second injury in the same cell. Irradiation at high dosage-rates causes arrest of mitosis so that the tumour goes into a resistant phase whereas low dosage-rates interfere much less with the progressive development of cells. High dosage-rates tend to produce many injuries in individual cells, often more than are required to kill it, but also to produce permanent injury in a greater number of cells than low-dosage rates. The effects due to variation in dosage-rate can also be obtained to some extent by fractionation of the dose (dividing the treatment into a number of exposures). Fractionation at high dosage-rates is particularly suitable for surface tumours where the radiation does not have to pass through normal tissues to reach the malignant growth. Low dosage-rates may be an advantage in the treatment of certain more deeply seated tumours where the radiation must pass through the skin, though more work on this subject is required before this can be established or disproved. The long cell cycle in the basal layers of the skin and the low dosage-rate both favour restitution of chromosome breaks and recovery of the skin from damage. The low dosage-rate will also produce less permanent injury to the tumour cells, but the relative effect for any given dose to the skin may be greater with certain types of tumour.

\section{Effect of Variations in the Dose-Time Relationship} on Tissue Reaction

We have seen that therapeutic doses of radiation affect primarily cells that are about to divide, the chief permanent result being degeneration and death of these cells manifested most clearly and most rapidly through mitosis. In many tumours adjacent cells frequently divide synchronously so that radiation-induced degeneration often involves small volumes of tissue scattered through the tumour mass. This irregular break-down of the $\frac{\text { O }}{\sigma}$ tumour results in a gross disturbance of the $\stackrel{\Xi}{2}$ organisation of the neoplasm as a whole, with 3 secondary changes, such as alterations in blood $\stackrel{\odot}{\circ}$ supply, which may lead to further degeneration.. . The body responds with an attempt at tissue repair, $\overrightarrow{\vec{F}}$ with infiltration of defence cells, digestion and removal of debris and the formation of fibrous? tissue. This normal tissue reaction plays an $\mathrm{im}-\frac{\bar{\sigma}}{\overline{0}}$. portant part in tumour regression and is initiated $\frac{\rho_{\sigma}}{\vec{\sigma}}$ by the radiation-induced break-down of cancer $\stackrel{D}{\circ}$ cells. Further treatment given too soon, when the majority of the dividing cells present have been $\vec{\circ}$ affected and the normal tissue reaction is at its height, may do more damage to the repair process $\vec{\omega}$ than to the tumour. Subsequent treatment given $\frac{P}{8}$ too late will either fail to maintain the rate of cello degeneration and so inhibit the normal tissue reaction, or allow the repair processes in parts of $N$ the tumour to progress too far before sufficient damage has been done to the cancer cells. Fibrous. $\vec{v}$ strands developing within the tumour substance $\vec{N}$ may cut off and protect islets of viable tumour cells

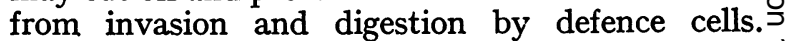
The timing of fractionated treatment may therefore $\vec{z}$ be an all important factor in the successful removal o of malignant tumours, particularly the more resistant ones, as incorrect timing may give the masss $\vec{A}$ an increased resistance to further irradiation.

\section{Radioresistance and Radiosensitivity}

There has been much confusion caused by the different interpretations placed on the term "radio-s sensitivity." There is a wide variation in cellular radiosensitiveness, "a dose of only $40 \mathrm{r}$. will kill ${ }^{-}$ half the individuals in a clutch of Calliphora eggs,,$\frac{3}{3}$ whereas a dose of $330,000 \mathrm{r}$. is required to produce 50 per cent of deaths in a culture of Colpidium" (Crowther). Bergonié and Tribondeau in 1906 stated that the radiosensitivity of cells varied 3 directly with their degree of proliferative activityo and inversely with their evolution from embryonal types and degree of differentiation. The response of a tumour to irradiation does not depend, however, solely on the type of cells of which it iso composed but also on the environment in which? it is growing - not only on histological structureo but also on site. The grouping of tumours histo logically in order of rate of immediate tumourer response to irradiation (the usual interpretation off radiosensitivity) gives little information as tow curability and takes no account of the variation in response that occurs within recognised histologicak groups or from one site to another. Squamous-? cell carcinomata of the buccal cavity undergoos complete regression following irradiation more frequently than their metastases in the cervicab lymph nodes, whereas the reverse is almost certainly, 
the case with adenocarcinomata of the breast and their deposits in the axillary nodes. Broders' grading of tumours according to their degree of differentiation is a valuable guide as to the probability of their early dissemination but, as has been shown by Spear and Glücksmann, provides no clear indication as to the likely response of the primary tumour to irradiation. In fact if we take "radiosensitivity" to mean a favourable response to radiation, it is often the more highly differentiated squamous-celled carcinomata that are the most radiosensitive.

A lymphosarcoma composed of a mass of rapidly dividing cancer cells with little supporting stroma will frequently show a dramatic initial response to treatment for with massive cell degeneration the tumour collapses, only too frequently to release viable cells to be carried to distant parts of the body. An osteogenic sarcoma composed of cells with a very different cycle and with a firmer supporting stroma will usually show no obvious immediate clinical response to treatment at all, but there is some evidence that the cancer cells themselves are not so insensitive to irradiation as might appear on superficial clinical observation. These two tumours require very different types of treatment in so far as the time-dose relationship is concerned. The tendency to deliver a higher dose in a shorter time the more resistant the tumour appears to be, is giving way to planned fractionation of the dose to suit the individual requirements of the tumour concerned according both to its histological structure and to the environment in which it is growing.

\section{(3) Radiotherapeutic Apparatus}

The Radiotherapist should have available a wide variety of apparatus designed to enable him to administer an adequate dose in the best possible way to suit each tumour and to concentrate this dose in the volume of tissue occupied by the tumour. For this purpose a large number of differing types of radium containers, radon seeds, teleradium units and X-ray tubes are required. In the future greater use will almost certainly be made of the Cyclotron either to provide artificial radioactive substances for injection or a beam of neutrons. Betatrons should provide new opportunities for the treatment of deep-seated cancer with very penetrating $X$-rays where the maximum energy absorption is below the surface and a high dose can be concentrated in the tumour more effectively than has been possible in the past.

\section{- Radium}

The gamma rays from radium are applied to the patient in one of three main ways. In the first method the radium is enclosed in needles or tubes usually made of platinum of sufficient thickness $\frac{}{2}$ to cut off the alpha and beta rays (Fig. Io). The $\frac{3}{\infty}$ amount of radium in each needle rarely exceeds $a_{c}$ few milligrammes and these needles are either: inserted into the patient's tissues or are mounted $\overrightarrow{\vec{F}}$ in an applicator of wax or other similar materialo which is fitted to the body surface. The needles: are left in position for the time necessary to given the required dose. Measurements of the radiation $\bar{\alpha}$ in the neighbourhood of these needles and tubeso and planned arrangement on the surface, in the ${ }^{\infty}$ tissues or in body cavities, has transformed thiso type of radium therapy which has recently becomefar more accurate and efficient chiefly as the result of the work of Paterson and Parker. The secondo general method is to have a considerable quantitys. of radium, up to several grammes, in a container and use this in the same way as an X-ray tube is used: This is known as a teleradium unit or radium "bomb" (Fig. II).

Teleradium units containing as much as Iov grammes are now in use and larger units still areo in preparation. It is probable that units of this type will in future contain tubes of artificial radio 3 active elements with a long half-value period Teleradium units have proved to be of value parti- $\overrightarrow{0}$ cularly in the treatment of some tumours situateds a short distance below the surface. This as especially so in the region of the head and neako where the distribution of the energy absorbed well suited to the difficult problem of applyinge multiple small fields to an irregular curved surface $\frac{0}{0}$

The third method is to use radon, a gas which is given off from radium, and to enclose it in smalb platinum or gold "seeds." These seeds can be inserted into the patient's body and left there for the radioactivity of radon disappears almos? completely after a few weeks (Fig. I2). Radiump needles on the other hand must never be left in. contact with the patient longer than the calculated time to give the required dose since gamma rays will continue to be given off indefinitely with no appreciable diminution in intensity (half-value period $\mathrm{I}, 65^{\circ}$ years).

\section{$X$-rays}

A radiotherapy department should be supplied with a number of $\mathrm{X}$-ray tubes generated at voltages varying from 50 kilovolts to 500 kilovolts or in some cases to I,000 kilovolts (Fig. I3) or morew This provides the radiotherapist with a range of beams of varying penetration to enable him to select the most appropriate apparatus for eacto patient with which he has to deal. Low-voltage apparatus with the source of the X-rays placed close to the skin is used for superficial lesions and advances have been made in design of apparatus? 
and application of such beams of X-rays by.Schaeffer, Witte, Chaoul, and Adam, this type of treatment being known as "Contact therapy" (Fig. I4).

In recent years great improvement has taken place in the accurate localisation in deep-seated tumours of the energy absorbed from external high-voltage X-rays and teleradium. This is a result of Mayneord's work on three-dimensional or volume distribution. An improvement in the results of treatment to some deep-seated tumours previously regarded as almost outside the scope of radiotherapy is now becoming manifest as the result of this work. With increased penetration, improved means of tumour localisation and more accurate beam direction, further improvement may be expected.

\section{Artificial Radioactivity}

The study of the properties of artificial radioactive substances is proceeding rapidly together with their application in the treatment of disease. They have a very important rôle to play in physiological research for radioactive atoms of various elements can be followed in their progress round the body and used as "tracers." Spear wrote, "one radioactive atom in a million is sufficient for the progress of the substance to be followed with extreme accuracy by physical detectors and at this dilution no biological effects of the radioactivity can be detected. . . . It is possible to make radioactive isotopes of all the stable elements, and these isotopes behave identically with the commonly obtained element both chemically and physiologically. ... Radio sodium, given to a patient by the mouth as common salt, can be detected by means of a Geiger counter within two minutes of administration in the finger tips." The production of fairly large quantities of rare stable isotopes of carbon $\left(\mathrm{C}^{13}\right)$ and nitrogen $\left(\mathrm{N}^{15}\right)$ which may be introduced into sugars or proteins and followed with the help of the mass spectrograph may also prove to be of great value in the study of metabolism.

In the treatment of malignant disease radioactive elements are being sought which will be selectively absorbed in certain tissues where radiation is required. It is already known that the concentration of radio iodine can be raised in animals to the point at which it completely destroys the thyroid gland without affecting the parathyroids. In man radio phosphorus ${ }_{15} \mathrm{P}^{32}$ (Fig. 5) which is absorbed selectively in the bone, bone marrow, spleen, lymph nodes, liver, and proliferating cells generally has been used for the treatment of the leukaemias, lymphadenopathies, and polycythaemia vera. Radio strontium which is selectively absorbed in bone is now undergoing clinical trial.

\section{(4) Uses and Limitations of Radio- therapy for Cancer}

Surgery and radiotherapy are still the only $\frac{1}{\infty}$ known means of curing cancer. Much interesting 2 work on chemotherapy is being done but despite the reports which appear in the lay press, the more sensational of which refer to the success of new $\overline{0}$ "cancer cures" and which are deprecated as much ㅇ by the serious workers in this field of research as by anyone else, there is still no real evidence $\frac{\widehat{\phi}}{\circ}$ that human cancer has yet been cured by such means. The outstanding success in palliation so ${ }^{\circ}$ far achieved by chemotherapy has been the use of $\overrightarrow{0}$ oestrogens in the treatment of cancer of the prostate. $\overrightarrow{\vec{H}}$

It is customary to regard cancer as a hopeless $\stackrel{\omega}{\omega}$ disease and for references to appear frequently to $\overrightarrow{0}$ the search for a "cure," as though no method of 3 curing any form of this condition was known, and as though it was a single disease entity requiring $\underset{\sim}{N}$ only some one magic drug for its elimination. $\triangleq$ This hopeless attitude towards what has been $\overrightarrow{\vec{v}}$ achieved and faith in some single "cure" that awaits us round the corner is by no means confined 9 to the lay public and is responsible for some of the $\lrcorner$ delay that occurs in sending patients for treatment 3 at an early stage of the disease while it is still curable by means already at our disposal. Cancer $\overrightarrow{0}$ at most sites in the body is curable if treated earlog. Even more is to be expected from improved methoo of early diagnosis and the recognition by the medical profession as a whole of the urgency of sending a patient with cancer to a centre where full modern facilities for treatment are available, $\frac{0}{\infty}$ than from the improvements that are taking place $\stackrel{\circ}{\circ}$ in the existing treatment methods. While new $\overrightarrow{0}$ discoveries related to the various predisposing 3 causes of cancer at different sites in the body, and the cause might well revolutionise treatment of malignancy within the cell, such advances may, for $\frac{}{3}$ all we know, be long delayed. In the meantime, while we wait for practical results to appear from $\frac{9}{3}$ the careful and patient research work that is being carried out, over 70,000 persons die from cancer in this country each year, many of them quite unnecessarily, because the available treatment that 9 could have cured them at one stage was either $D$ applied too late or not applied at all.

A woman with a lump in the breast requires N investigation to exclude cancer, and treatment for cancer if the diagnosis is established, as urgently ? as a patient with acute appendicitis or a per- $\omega$ forated gastric ulcer requires operation. To take two extreme examples: (I) Patients who worke with pitch and tar are liable to skin cancer but the risk is appreciated, they are examined at regular? intervals by their industrial medical officers and $\underset{T}{0}$ trained to report the smallest skin lesions. The $\frac{\overrightarrow{0}}{\mathrm{D}}$ 
symptom-free rate in this group of patients with adequate treatment is Ioo per cent. (2) Cancer of the lung on the other hand has an insidious onset starting as a rule with a dry cough, often of a different character to any cough that the patient may have had previously, but causing him no alarm at first so that he seldom reports it to his doctor until he either develops pain, feels weak and tired, or has an haemoptysis. If he does report early he is often reassured and given a bottle of medicine. Even if X-rayed in the early stages he is all too frequently merely told to come back in a month or two for a repeat examination either because the shadow was not large enough to leave no doubt as to its nature radiologically or because the radiograph showed no abnormality. By the time that a bronchoscopy is first performed the great majority are inoperable and most of the remainder beyond the stage when radiotherapy can achieve more than temporary palliation. In a recent review of $I_{50}$ consecutive new cases of cancer of the lung seen in one year at the joint consultation clinic held by members of the staffs of the Brompton Hospital for Diseases of the Chest and the Royal Cancer Hospital it was found that the average delay in reporting the first symptom to a doctor was 3.5 months, and the average delay from this first consultation till the patient came for treatment was a further 5 months: 8.5 months average delay, the greater part of which was directly or indirectly the responsibility of the medical profession.

Surgery and radiotherapy are partners in the treatment of cancer; in some cases either alone will produce comparable results, in others one is superior, and in a third group the patient's best prospect lies in a planned combination of the two. We are here concerned with the rôle of radiotherapy but it is wrong to regard this method of treatment in isolation. Radiotherapy is now well established, its field is becoming better defined and is expanding as knowledge and improved technical facilities increase. Advances in accuracy of application have removed most of its dangers in experienced hands and further improvement in results is confidently to be expected.

\section{Radiotherapy for Accessible Cancer}

The outstanding achievements of radiotherapy have been in the treatment of accessible cancer. It plays the paramount part in the treatment of cancer of the skin. A symptom-free rate of over 90 per cent is obtained with adequate treatment for basal-cell carcinomata and with the help of surgery in the more resistant cases a disease-free rate of Ioo per cent should be obtained. With squamous-cell carcinoma of the skin the position is complicated by the liability to metastases, but the local primary tumour should be eradicated ire nearly every case and in combination with surgerya a 70-80 per cent symptom-free rate can be achievedk even with the present far too high proportion of late cases that are coming to hospital.

Cancer of the lip and mouth are now pre $\vec{F}$ dominantly the province of the radiotherapist? A judicious selection of treatment method, frome interstitial radium implants and contact $\mathrm{X}$-ray therapy for the primary sometimes combined with block dissection of the neck, to X-rays and tele radium for both the primary and the neck nodesw in the later cases, produces. a five-year survival- 0 rate of approximately 65 per cent in early cases. and one of over 30 per cent in all cases treated $\vec{\omega}$ Such results are, however, only obtained at some of the larger centres, notably at the Christio Hospital and Holt Radium Institute in Manchester?

Intrinsic carcinoma of the larynx is being treated by radiotherapy with increasing frequency and is displacing surgery, despite the fact that brilliant successes have been achieved by this means $\vec{N}$ largely because the results of radiotherapy are comparable with those of surgery in the earlier cases, are superior in the later ones and the mutila tion of total laryngectomy is avoided. Contacto $\mathrm{X}$-ray therapy after removal of part of the thyroid cartilage (a good example of the increasing use rof surgery to give access for radiotherapy) is employed, but the majority of these cases are now treated 8 . $\mathrm{X}$-rays applied externally or by teleradium whele seems to have several advantages in this situation Lederman has recently published an account of 23 operable cases of intrinsic cancer of the larynx treated by teleradium at the Royal Cancer Hospita in which 18 were alive one year after treatment 3 and 5 of which had lived 5 years or more. Other malignant tumours of the pharynx and larynx ares almost exclusively treated by external irradiation with variable success but with definite improve 3 . ment over the past ten years.

Cancer of the breast is now recognised as being the joint concern of both surgeons and radios therapists neither alone being able to achieve results comparable with those resulting fromo combined treatment. McWhirter at the Roya? Infirmary, Edinburgh has shown that the symptom? free rate in cases with no evidence of lymphnode involvement can be raised to 90 per cent by comor bined treatment, and that where lymphnodes are involved the symptom-free rate can be more thanu doubled if X-ray treatment is added to surgery; o 50 per cent symptom-free rate being obtained in those operable cases which are found to have metastasised to lymphnodes.

Miss Hurdon will be long remembered for her work in the radium treatment of cancer of the cervix uteri at the Marie Curie Hospital where 
five-year survival-rate of 80 per cent was obtained in Stage I cases and of 35 per cent in all cases seen. Radium applied to the uterus and vagina combined with external high voltage X-rays based both on scientific principles of distribution of the energy absorbed and on the biological response of individual tumours as recorded, for instance, by Spear and Glücksmann at the Strangeways Laboratory in Cambridge should improve these results still further.

\section{Radiotherapy for Deep-Seated Cancer}

With deep-seated cancer the results of radiotherapy are still far from satisfactory but notable advances have been made in recent years. The technical problems of administration of adequate treatment are greatly increased when the tumour is situated at a depth, but there is evidence that these can now be overcome to a great extent.

Cancer of the oesophagus is still rarely cured by any means, though Guisez in Paris obtained some good results with a radium bougie. An account of three five-year survivals following external highvoltage X-ray therapy has been published from the Royal Cancer Hospital. Cancer of the stomach and intestine is almost the exclusive province of the surgeon. Surgery has many cures of cancer of the rectum to its credit, a number of these patients being alive and well 20 years or more after treatment. The use of very high voltages has already brought about an improvement in the $\mathrm{X}$-ray treatment of the more advanced cases of cancer of the rectum, the work of Phillips with the million-volt X-ray therapy plant at St. Bartholomew's Hospital being outstanding in this field.

In the urogenital tract surgery is the method of choice in most cases though the improvement in the treatment of cancer of the bladder by means of radon seed implants or external high-voltage $\mathrm{X}$-rays is giving radiotherapy a prominent place in the treatment of tumours at this site.

Cancer of the lung presents an exceptionally interesting problem both on account of the apparent increase in frequency of this disease and the excellent results obtained in a few cases by pneumonectomy. The number of patients suitable for this operation is, however, so small that a concentrated attack upon this disease by means of X-rays is being undertaken. Little success was achieved with the old methods but an improved method of $\mathrm{X}$-ray treatment adopted over the past two years at the Royal Cancer Hospital has shown that extremely valuable palliation can be achieved and that a number of patients can be returned to work free from symptoms. It will be of great interest to see if this freedom from symptoms will be maintained in a reasonable proportion of the cases so treated.

The connective tissue sarcomata have been regarded in the past as radioresistant but this conclusion was reached mainly on a basis of the immediate clinical response, and further work is $\frac{3}{\circ}$ required before they are lightly dismissed as beyond $\stackrel{\circ}{ }$ the scope of radiotherapy. There are those who:maintain that a combination of irradiation and $\overrightarrow{\vec{m}}$ removal of the limb or tissues affected offers thebest prospect of success. It is to be hoped that $a \frac{\bar{\sigma}}{\sigma}$ group of workers will combine to investigate the response of these tumours to the various com- $\bar{\Phi}$ binations of treatment method at present available, as there is some evidence that more could ${ }^{\infty}$ be done for these patients than is being achieved $\overrightarrow{0}$ at present. Too few cases are seen in any one $\overrightarrow{\vec{H}}$ centre for a planned research programme to be ${ }_{0}^{\omega}$ carried out in a reasonable time.

The lymphadenopathies and leukaemias are 3 treated by radiotherapy and useful palliation usually obtained, though no real progress towards curing these diseases has yet been made. This would seem to be one of the fields where the use:of radiotherapy as a means of relieving symptoms $N$ and improving the patient's general condition mayo well be combined in the future with attempts at $a_{\triangle}$ more fundamental attack upon the disease process 3 by means of chemotherapy or hyperpyrexia. The intriguing prospect of the introduction of arti- $\overrightarrow{0}$ ficially radioactive elements into compounds wi known growth inhibitory action is one which might possibly link chemotherapy and radiothera) together in an attempt to deal with this group of fatal diseases.

There are a number of other sites where radio-屰 therapy has a part to play in the treatment of malignant disease, such as the thyroid, brain, $\overrightarrow{0}$ and salivary glands, for instance, but no more 3 than an indication of the scope of radiotherapy can be given here. As a means of palliation in? advanced cancer and particularly in the relief of pain, radiotherapy is the most valuable therapeutic method at our disposal. Its place in cancer? treatment would be assured on these grounds alone even if it were not successful in curing a singleo patient with this disease. Advances in apparatus construction and the availability of artificial radio을 active substances, together with increasing know- $\rightarrow$ ledge of the response of individual tumours and the tissues around them to irradiation holds outur exciting prospects for the radiotherapist. The radiotherapist would, however, gladly postpone thes acquisition of these long awaited additions to hiș department in exchange for a few months off the average length of history given by the patientso with cancer who come for treatment. He wouldo improve his results more by decreasing the interva ${ }^{\text {f }}$ between patients' first visits to their doctors ando their first treatment than by adding any costly new piece of apparatus to his existing equipment. 
Summary

Radiotherapy is the use of some ionising radiations in medicine to produce a biological effect, either in the form of particles or waves of sufficient energy to produce the effect desired. These radiations are obtained from radioactive elements undergoing spontaneous disintegration (e.g. radium or radon); from elements previously excited which, when reverting to their normal state, give off the energy acquired (e.g. radioactive phosphorus, or strontium) from X-ray tubes where metals (e.g. tungsten) are bombarded with electrons; or from apparatus either designed to accelerate heavy particles and produce a beam of neutrons (Cyclotron) or to accelerate electrons, which may be used directly, but more probably to produce very high-voltage $\mathrm{X}$-rays (Betatron). The absorption of energy from these radiations results in intense electronic activity in the tissues.

The amount of damage done depends on the amount and distribution of the energy absorbed, $\stackrel{\oplus}{\bullet}$ the rate of absorption, the intervals between exposures, the total treatment period, the sensi-z tivity of the cells, and the nature and state of the ${ }_{\stackrel{\mathbb{D}}{ }}$ tumour bed. A wide variety of apparatus is. necessary for the efficient treatment of all types of $\Rightarrow$ case. The results of treatment with early acces- sible cancer are excellent and with deep-seated? cancer are improving, and may be expected to $\frac{\bar{N}}{\overline{0}}$ improve considerably in the future. Earlier? diagnosis and treatment and more knowledge of the ${ }^{\circ}$ biological effects are now more urgently neededœ than technical advances on existing lines.

\section{Acknowledgement}

This paper is based on a short series of lectureso given to medical students at the Royal Free Hospital. I am indebted to Dr. P. C. Koller and to Mr. L. F. Lamerton for the helpful advice they gave me in their preparation.

\section{THE TREATMENT OF SUPPURATIVE OTITIS MEDIA*}

\section{By S. E. Birdsall, F.R.c.s.}

My subject deals with suppurative otitis media, acute or chronic, and from the clinical aspect it comprises the majority of patients who present themselves to the practitioner with discharging ears.

It is the practitioner's first duty to decide whether the discharge is from the external meatus or from the middle-ear, and it is obvious that no treatment can logically be prescribed until this distinct point has been settled.

It is an unfortunate fact that the present medical curriculum provides inadequate instruction in many so-called special subjects. Any general practitioner is certain to meet in his practice with many patients suffering from otorrhoea in the course of a year, and they will certainly be greater in number than those whom he finds with appendicitis or carcinoma of the alimentary canal, yet in his studentship he will have gained very scanty knowledge of the common condition of otitis media, compared with his intimate knowledge of the far rarer abdominal diseases.

Confronted with a patient who has a discharging ear, it is essential to know the origin of the discharge; is the diagnosis one of otitis externa or of otitis media? The distinction may be difficult. The following points may be helpful.

* Based on a lecture at The Memorial Hospital, S.E.I8, on 9.3 .46 .
First.-In otitis externa, tenderness is marke and pain may be slight. In otitis media, pain mo be very severe, and tenderness of the pinna invariably absent. Mastoid tenderness may be $\bar{\partial}$ present in either condition.

Secondly.-The character of the discharge affords $\frac{0}{\Phi}$ valuable evidence. In amount, it is more copious in otitis media. All discharge must first be removed by mopping or syringing. If it rapidly reappears, then we are probably dealing with otitis media, in which the secretion in the meatus is the overflow from an infected cavity lined with 3 mucous membrane. In otitis externa, the secretion is derived from the skin lining the meatus, $\underline{3}$. and it will not be replaced in a shorter time than an hour. If the discharge contains mucus, then it must come from the middle-ear as there are noo mucous glands in any skin. The discharge in? otitis externa is often bright yellow, as though stained with flavine.

Thirdly.-The meatus is usually bealthy in otitis media. In otitis externa there is usually $N$ some stenosis and the meatus may be very narrow owing to swelling of the lining skin, which either itches or is acutely tender.

Fourthly.-In a disease affecting the ear, no examination is complete unless the hearing be $\stackrel{\oplus}{\oplus}$ tested. It is essential first to clean the meatus ? of discharge. In otitis externa the hearing will ${ }^{\circ}$ be normal or but slightly diminished. In otitis $\underset{D}{0}$ 